\title{
Homology of spaces of knots in any dimensions
}

\author{
By V. A. VAssilieV \\ Steklov Mathematical Institute and Independent University of Moscow, \\ Gubinka 8, Moscow 117966, Russia
}

I shall describe the recent progress in the study of cohomology rings of spaces of knots in $\mathbb{R}^{n}, H^{*}\left(\left\{\right.\right.$ knots in $\left.\left.\mathbb{R}^{n}\right\}\right)$, with arbitrary $n \geqslant 3$. 'Any dimensions' in the title can be read as dimensions $n$ of spaces $\mathbb{R}^{n}$, as dimensions $i$ of the cohomology groups $H^{i}$, and also as a parameter for different generalizations of the notion of a knot.

An important subproblem is the study of knot invariants. In our context, they appear as zero-dimensional cohomology classes of the space of knots in $\mathbb{R}^{3}$. It turns out that our more general problem is never less beautiful. In particular, nice algebraic structures arising in the related homological calculations have equally (or maybe even more) compact description, of which the classical 'zero-dimensional' part can be obtained by easy factorization.

There are many good expositions of the theory of related knot invariants. Therefore, I shall deal almost completely with results in higher (or arbitrary) dimensions.

\section{Keywords: operads; order complexes; simplicial resolutions; combinatorial formulae; spectral sequences}

\section{Main construction}

We consider both the standard compact knots, i.e. smooth embeddings $S^{1} \rightarrow \mathbb{R}^{n}$, and the long knots, i.e. embeddings $\mathbb{R}^{1} \rightarrow \mathbb{R}^{n}$ coinciding with a standard linear embedding outside some compact subset in $\mathbb{R}^{1}$ (see figure 1 ).

The study of the latter space is more essential, because the algebraic structure of the cohomology ring of the space of standard knots is built from that of the similar ring for long knots (here playing the role of the 'coefficient ring'), the topological non-triviality of the circle $S^{1}$, and a certain family of its configuration spaces.

Let us denote by $\mathcal{K}$ the space of all smooth maps $S^{1} \rightarrow \mathbb{R}^{n}$ (respectively, of maps $\mathbb{R}^{1} \rightarrow \mathbb{R}^{n}$ with such boundary conditions). This is a linear (respectively, an affine) space. The discriminant $\Sigma \subset \mathcal{K}$ is the set of all maps which are not smooth embeddings, i.e. have either self-intersections or singular points. The space of knots is the difference $\mathcal{K} \backslash \Sigma$.

\section{(a) Arnold's reduction}

It is convenient to study the cohomology group of the space of knots by a sort of Alexander duality,

$$
\tilde{H}^{i}(\mathcal{K} \backslash \Sigma) \simeq \bar{H}_{n \infty-i-1}(\Sigma) .
$$

The bar in the notation $\bar{H}_{*}$ means that we consider Borel-Moore homology, i.e. the homology group of the one point compactification, and $n \infty$ is the notation for 


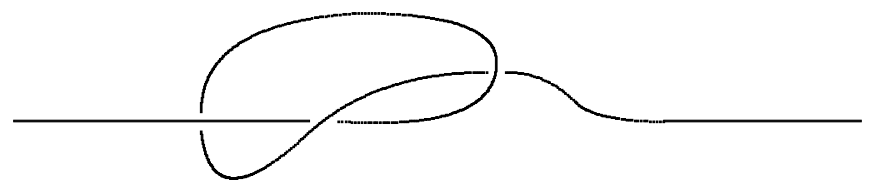

Figure 1. A long knot.
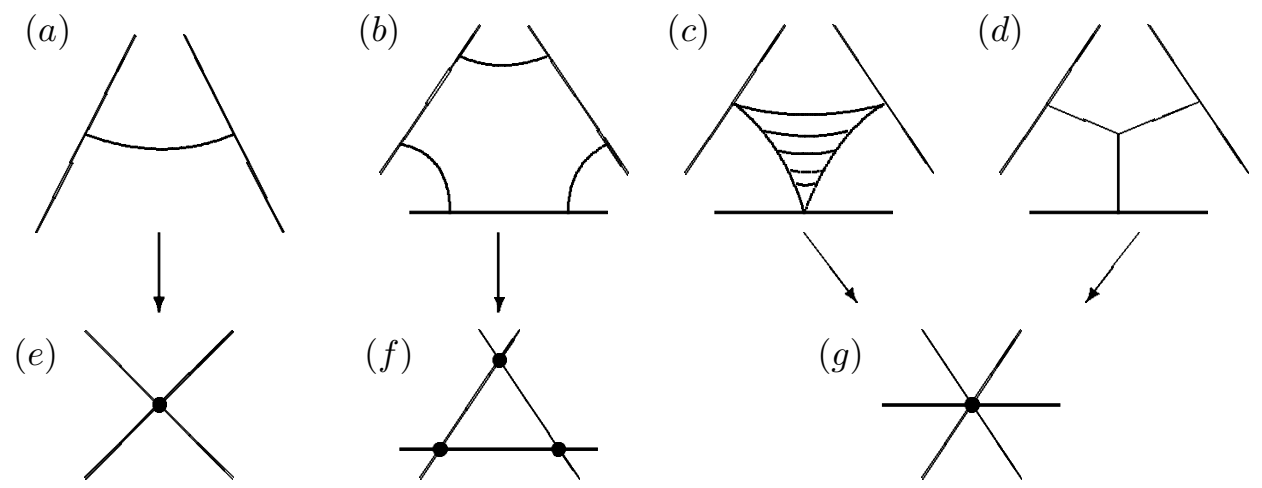

Figure 2. Simplicial resolutions of line arrangements.

the dimension of $\mathcal{K}$. Of course, the whole right-hand side in (1.1) is, strictly speaking, senseless. However, it can be given some strict sense by means of appropriate finite-dimensional approximations to the space $\mathcal{K}$ (see $\S 1 h$ ). Roughly speaking, the elements of this group are the semialgebraic cycles of codimension $i+1$ in $\mathcal{K}$. A reduction like (1.1) was used first by Arnold $(1970,1989)$ (in the finite-dimensional situation of the standard discriminant varieties in the space of polynomials in $\mathbb{C}^{1}$ or $\mathbb{R}^{1}$ ), and is very useful in the whole theory of discriminants. Indeed, the discriminant sets of singular maps are singular varieties, stratified in the correspondence with the classifications of (multi)singularities, and, as we shall see in our special case, a lot of their topological properties can be expressed in terms of these stratifications.

\section{(b) Simplicial resolutions}

Further, it is convenient to study the topology of discriminants by means of the simplicial (or, more generally, conical) resolutions. These resolutions provide topological spaces homotopically equivalent to initial ones (in particular having the same homology groups), but having a more transparent homological structure, which is easier to calculate. An important illustration of this method comes from the theory of plane arrangements.

Let us consider a finite collection of affine planes (of arbitrary dimensions) in $\mathbb{R}^{m}$,

$$
L=\bigcup_{i=1}^{N} L_{i},
$$

and suppose that we need to calculate the cohomology group of its complement $\mathbb{R}^{m} \backslash L$ (or, equivalently, the Alexander dual group $\bar{H}_{*}(\Sigma)$ ). 


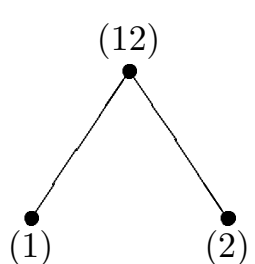

(2)

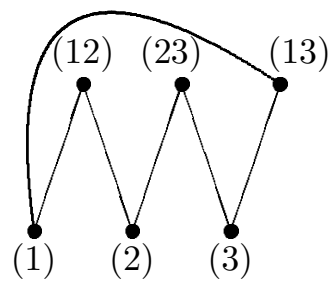

(3)

(13)

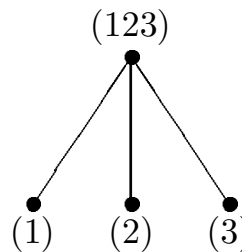

Figure 3. Order complexes for line arrangements.

The resolutions of three line arrangements shown in figure $2 e-g$ are given in figure $2 a-d$. At the first step we take these lines separately, and then add some furniture, spanning the points of the separated lines arising from one and the same point below. For figure $2 e, f$, all standard constructions of simplicial resolutions give essentially one and the same space. Namely, if we have a double intersection point of the arrangement, then we mark the corresponding two points on separated lines and join them by a segment. However, there are two main different ways to resolve the arrangement of figure $2 g$.

One of these ways (modelling the combinatorial formula of inclusions and exclusions) will first join by segments all pairs of intersection points of any two planes, independently of whether these points belong to some planes more than to the initial planes. Then, over all triple-intersection points of the arrangement, we obtain a triple of segments, forming a triangle 'without the interior part', which will be filled at the next step. At the next step, the preimages of quadruple points (if any) will be filled by tetrahedra, etc. We shall call this resolution (and its generalizations) the naive resolution, in contrast to the economical one, which provides part $(d)$ of figure 2 and is based on the notion of the order complex of a partially ordered set (= poset) (see, for example, Deligne 1972).

Definition 1.1. Given a poset $(A,>)$, the corresponding order complex $P(A)$ is the simplicial complex, whose vertices are the points of the set $A$, and whose simplices span all the sequences of such points monotone with respect to the partial order.

An important family of posets is provided by the theory of plane arrangements. Given such an arrangement (1.2), for any subset $I$ of the set of indices $\{1, \ldots, N\}$, denote by $L_{I}$ the plane $\bigcap_{i \in I} L_{i}$. All planes of the form $L_{I}$ are (not canonically) called the strata of the arrangement $L$. The set of all strata is a poset (by inclusion). Let $P(L)$ be the corresponding order complex. The order complexes of three arrangements of figure 2 are shown in figure 3. Here, the vertex labelled by (12) denotes the intersection plane (point) of the first and the second planes (lines) labelled by (1) and (2), respectively.

The economical simplicial resolution of the arrangement $L$ will be defined as a subset of the direct product $P(L) \times \mathbb{R}^{m}$. For any non-empty stratum $L_{I}$, let $\Delta(I) \subset P(L)$ be the order subcomplex subordinate to $L_{I}$, i.e. the subcomplex of $P(L)$ consisting of only those simplices all of whose vertices correspond to planes containing $L_{I}$ (or coinciding with $L_{I}$ ). This is a compact contractible space. Indeed, all its maximal simplices have the common vertex $\left\{L_{I}\right\}$. Then the resolved arrangement $\tilde{L}$ is defined by the formula

$$
\tilde{L}=\bigcup\left(\Delta(I) \times L_{I}\right) \subset P(L) \times \mathbb{R}^{m},
$$

with union over all geometrically distinct strata $L_{I}$. 
For figure $2 e, f$, the corresponding graphs drawn above them can be considered as pictures of such resolutions as well, especially if we distinguish the middle points of the inserted segments. These segments can be considered as products $\Delta(I) \times L_{I}$ for two-element sets $I=(i, j)$, so that $L_{I}$ are the intersection points of any two lines $L_{i}$, $L_{j}$, and the order subcomplex $\Delta(I)$ is the union of two segments joining the vertex $(i, j)$ to two vertices $(i),(j)$, corresponding to these two lines.

The obvious projection $P(L) \times \mathbb{R}^{m} \rightarrow \mathbb{R}^{m}$ defines a map $p: \tilde{L} \rightarrow L$. This map is proper and semialgebraic, and all its fibres are different spaces of the form $\Delta(I)$. Therefore, it is a homotopy equivalence. Moreover, its extension to the map of onepoint compactifications

$$
\overline{\tilde{L}} \rightarrow \bar{L}
$$

is also a homotopy equivalence, defining, in particular, an isomorphism of BorelMoore homology groups. But why is the resolved space $\tilde{L}$ better than the initial one?

\section{(c) The filtration}

There is a natural increasing filtration,

$$
F_{1} \subset \cdots \subset F_{n-1}=\tilde{L},
$$

on the resolved space $\tilde{L}$. Its term $F_{p}$ equals the union as in (1.3) but only over the strata of codimension less than or equal to $p$. The difference $F_{p} \backslash F_{p-1}$ is the union of products $\check{\Delta}(I) \times L_{I}$ over all strata $L_{I}$ of codimension exactly $p$, where $\check{\Delta}(I)$ is equal to $\Delta(I)$ less the link $\partial \Delta(I)$ of $\Delta(I)$, i.e. the union of simplices not containing the minimal vertex $\left\{L_{I}\right\}$. Indeed, the set $\partial \Delta(I) \times L_{I}$ belongs to the lower term $F_{p-1}$ of the filtration. This filtration can be extended to a filtration $\left\{\bar{F}_{0} \subset \bar{F}_{1} \subset \ldots\right\}$ of the compactification $\tilde{L}$. Its term $\bar{F}_{0}$ consists of the added point, and other terms $\bar{F}_{p}$ are just the closures of the similar terms of the filtration on $\tilde{L}$.

Theorem 1.2. This filtration homotopically splits into the wedge of corresponding quotient spaces, i.e. there is a homotopy equivalence

$$
\overline{\tilde{L}} \sim \bar{F}_{1} \vee\left(\bar{F}_{2} / \bar{F}_{1}\right) \vee \cdots \vee\left(\bar{F}_{N-1} / \bar{F}_{N-2}\right) .
$$

This theorem was proved in Ziegler \& Živaljević (1993). For an equivalent (and simultaneously obtained) result in terms of the 'naive' resolution, see Vassiliev (1993).

In particular, we have the splitting of the Borel-Moore homology group of $\bar{L}$ (or, which is the same by the Alexander duality, of the cohomology group of $\mathbb{R}^{m} \backslash L$ ):

$$
\begin{aligned}
H^{m-i-1}\left(\mathbb{R}^{m} \backslash L\right) & \simeq \bar{H}_{i}(\bar{L}) \equiv \bar{H}_{i}(\overline{\tilde{L}}) \\
& \simeq \oplus \bar{H}_{i}\left(\check{\Delta}(I) \times L_{I}\right) \equiv \oplus H_{i-\operatorname{dim} L_{I}}(\Delta(I), \partial \Delta(I)),
\end{aligned}
$$

where $\tilde{H}_{*}$ denotes the homology group reduced modulo a point, and summation is over all strata $L_{I}$ of the arrangement.

This expression was obtained first by Goresky \& MacPherson (1988) by a different method. It implies that the homology groups of $\mathbb{R}^{m} \backslash L$ are completely determined by dimensions of spaces $L_{I}$.

The splitting (1.5) implies that even the stable homotopy type of this complementary space depends only on these data. 


\section{(d) Geometrical interpretation}

Formula (1.6) has the following direct realization (Ziegler \& Živaljević 1993; see the present formulation in Merkov 2001). Suppose a Euclidean metric is fixed in $\mathbb{R}^{m}$. Consider a constant vector field $V$ ('power') in $\mathbb{R}^{m}$ in the general position with respect to $L$. For any $k$-dimensional simplex of the order subcomplex $\Delta(I) / \partial \Delta(I)$ (i.e. for a decreasing sequence of $k+1$ strata $L_{I_{1}} \supset L_{I_{2}} \supset \ldots \supset L_{I_{k}} \supset L$ ) and for any point $x \in L_{I}$, consider the sequence of $k+1$ rays in $\mathbb{R}^{m}$ issuing from $x$, namely, the trajectories of $x$ in the planes $\mathbb{R}^{m}, L_{I_{1}}, \ldots, L_{I_{k}}$ under the action of this power. (If we realize $V$ as the gradient field of a generic linear function $\theta: \mathbb{R}^{m} \rightarrow \mathbb{R}$, then these rays will be the trajectories of gradients of restrictions of $\theta$ to these planes.) As $V$ is in the general position with respect to $L$, these rays are linearly independent, and their convex hull is linearly homeomorphic to a $(k+1)$-dimensional octant with origin at $x$. Such octants over all $x \in L_{I}$ sweep out an $\left(i+1+\operatorname{dim} L_{I}\right)$-dimensional wedge in $\mathbb{R}^{m}$.

If we have a cycle $\alpha$ of the complex $\Delta(I) / \partial \Delta(I)$, then the sum of (uniformly oriented) corresponding wedges is a relative cycle in $\mathbb{R}^{m}(\bmod L)$, and the relative homology class $\nabla \alpha$ of the latter cycle depends only on the class of $\alpha$ in $H_{*}(\Delta(I), \partial \Delta(I))$.

Finally, we take the class in $H^{*}\left(\mathbb{R}^{m} \backslash L\right)$ Poincaré-Lefschetz dual to $\nabla \alpha$ in $\mathbb{R}^{m} \backslash L$, i.e. defined by intersection indices with the relative cycle $\nabla \alpha$.

This realization depends on the choice of the direction $V$, but not very much. Two elements in $\bar{H}_{*}\left(\mathbb{R}^{m}, L\right)$, corresponding in this way to one and the same class $\alpha \in H_{*}(\Delta(I), \partial \Delta(I))$ via different generic functions, can differ only by elements of lower filtration, i.e. by a sum of similar classes coming from the summands $H_{*}(\Delta(J), \partial \Delta(J))$ corresponding to planes $L_{J}$ strictly containing $L_{I}$.

Moreover, if all strata $L_{I}$ have codimensions greater than or equal to 2 in all greater strata $L_{J}$, then the isomorphism (1.6) is canonical. In this case, the space of generic (in the desired sense) vectors $V$ is path-connected.

By analogy with knot theory, such realizations of elements of $H^{*}\left(\mathbb{R}^{m} \backslash L\right)$ can be called their combinatorial expressions.

\section{(e) Multiplication in cohomology}

Unfortunately, the usual homotopy type of the complement of an arrangement cannot be determined by the dimensional data.

The most developed case is that of complex hyperplane arrangements. In this case the multiplicative structure of the integral cohomology ring of the complement is determined by the dimensional data. The direct expression was obtained by Orlik \& Solomon (1980) with the help of some ideas from pioneering works of Arnold (1969) and Brieskorn (1973). However, even in this case (and even for central, i.e. passing through the origin, arrangements in $\mathbb{C}^{3}$ ), the fundamental group of the complement is not determined by these data. There exist pairs of arrangements with equal dimensions of all strata but with different fundamental groups (see Rybnikov 1998).

For arbitrary (not hyperplane) complex arrangements, the cohomology ring of the complement is also defined by the dimensional data. In the case of rational coefficients, this was proved in De Concini \& Procesi (1995), and, in the more complicated integral case, in Deligne et al. (2000) and de Longueville \& Schultz (2001) with the help of some ideas from Yuzvinsky (1998). 
Still, something good can be said even in the most general case of an arbitrary arrangement of real affine planes of arbitrary (possibly different) dimensions in $\mathbb{R}^{m}$. The graded ring associated with the filtered ring $H^{*}\left(\mathbb{R}^{m} \backslash L\right)$ is also defined by dimensional data and by some information on mutual orientations of all planes $L_{I}$.

Indeed, the splitting (1.6) is not canonical. The summands in the second line of (1.6) related to some stratum $L_{I}$ define well some elements of the first line only up to lower terms of the filtration. More precisely, they only define up to elements of similar terms $L_{\tilde{I}}$ with

$$
L_{\tilde{I}} \stackrel{\neq}{\supset} L_{I}
$$

However, let us rewrite equation (1.6) as that for associated graded groups:

$$
G r H^{*}\left(\mathbb{R}^{m} \backslash L\right) \cong \oplus H_{m-*-1-\operatorname{dim} L_{I}}(\Delta(I), \partial \Delta(I)) .
$$

The splitting in this formula is already canonical (up to the choice of orientations of planes $L_{I}$ ), and the multiplication in the associated graded ring is as follows.

Let us consider two strata $L_{I}, L_{J} \subset L$ and two cycles $A, B$ of the quotient complexes $\Delta(I) / \partial \Delta(I)$ and $\Delta(J) / \partial \Delta(J), \operatorname{dim} A=u, \operatorname{dim} B=v$, represented by linear combinations of simplices of subcomplexes $\Delta(I), \Delta(J)$ with boundaries only in $\partial \Delta(I)$ and $\partial \Delta(J)$. The shuffle product $A \odot B$ of these cycles is defined as follows (see Yuzvinsky 1998).

If $L_{I}$ and $L_{J}$ are not transversal (i.e. belong to some proper plane in $\mathbb{R}^{m}$ ) or have no intersection points, then $A \odot B=0$. Now suppose that $L_{I}$ and $L_{J}$ are transversal and $L_{K}=L_{I} \cap L_{J} \neq \emptyset$ (we can take $K=I \cup J$ ). Let $a \subset A$ and $b \subset B$ be some two simplices with $u+1$ and $v+1$ vertices, respectively, i.e. some decreasing sequences of strata of $L$ having $\left\{L_{I}\right\}$ and $\left\{L_{J}\right\}$ as their last elements. Consider all

$$
\left(\begin{array}{c}
u+v+2 \\
u+1
\end{array}\right)
$$

possible shuffles of these sequences, i.e. all (non-monotone) sequences of $u+v+$ 2 strata in which all elements of $a$ and $b$ appear, preserving their orders in the sequences $a$ and $b$. To any such shuffle, a monotone sequence corresponds: any element $\lambda$ of the shuffle coming from the sequence $a$ (respectively, $b$ ) should be replaced by the intersection of the corresponding stratum with the last stratum coming from the sequence $b$ (respectively, $a$ ) and staying before $\lambda$ in the shuffle. The obtained monotone sequence is, by definition, a $(u+v+1)$-dimensional simplex of the order complex $L_{K}$. The shuffle product of our simplices $a$ and $b$ is defined as the sum of all such simplices taken with signs equal to parities of the corresponding shuffles (i.e. the numbers of transpositions reducing them to the simple concatenation of sequences $a$ and $b$ ) multiplied by one sign more, which depends only on multi-indices $I, J$ and $K$, and which is defined by the comparison of the fixed coorientation of the plane $L_{K}$ in $\mathbb{R}^{m}$ with the ordered pair of coorientations of $L_{I}$ and $L_{J}$. The shuffle product of cycles $A$ and $B$ is defined by linearity. It is a relative cycle defining an element of the summand in the right-hand part of (1.7) corresponding to the stratum $L_{K}$. This element depends only on homology classes of $A$ and $B$ in the summands corresponding to $L_{I}$ and $L_{J}$.

Theorem 1.3 (see Yuzvinsky 1998, 1999; Feichtner \& Ziegler 2000; Deligne et al. 2000; de Longueville \& Schultz 2001). The isomorphism (1.7) 
commutes the shuffle product in its right-hand part and the multiplication in its left-hand part obtained from the usual cohomological multiplication. If all strata $L_{I}$ have codimensions greater than or equal to 2 in all greater strata $L_{J}$, then the same is true for the isomorphism (1.6) and the multiplication in the ring $H^{*}\left(\mathbb{R}^{m} \backslash L\right)$ itself, and not only in its graded ring.

This is a corollary of the explicit construction described in $\S 1 d$. Given two strata $L_{I}, L_{J}$ and classes $\alpha \in H_{*}(\Delta(I), \partial \Delta(I)), \beta \in H_{*}(\Delta(J), \partial \Delta(J))$, we can realize corresponding elements in the left-hand part of (1.6) with the help of directions $V_{I}$, $V_{J}$ in $\mathbb{R}^{m}$ that are in general position if $L_{I}$ and $L_{J}$ have non-empty transversal intersection. If not, then these directions should be opposite to one another and transversal to a plane separating or containing these strata.

\section{(f) All the same in the space of curves}

The discriminant in the space of curves $\mathcal{K}$ is also a union of planes. For any pair of points $a, b$ in $\mathbb{R}^{1}$, we consider the plane $L(a, b) \subset \mathcal{K}$ consisting of all maps $f: \mathbb{R}^{1} \rightarrow \mathbb{R}^{n}$ such that $f(a)=f(b)$ if $a \neq b$ or $f^{\prime}(a)=0$, if $a=b$. Any point of the discriminant belongs to at least one such plane. Then we take the order complex of all possible intersections

$$
L\left(a_{1}, b_{1}\right) \cap L\left(a_{2}, b_{2}\right) \cap \cdots
$$

and limit positions of such intersections (all of which are affine planes in $\mathcal{K}$ whose codimensions are multiples of $n$ ), supply this order complex with a natural topology, and define the simplicial resolution in exactly the same way as previously, i.e. as a subset of the direct product of this order complex and the space $\mathcal{K}$. Then we define the filtration on this resolution by the codimensions (divided by $n$ ) of these planes and consider the arising spectral sequence.

A unique serious difficulty here appears from the fact that some points of $\Sigma$ belong to infinitely many planes $L(a, b)$. For instance, a map $f$ sends a segment of $\mathbb{R}^{1}$ into one point or sends two segments of $\mathbb{R}^{1}$ into one and the same arc in $\mathbb{R}^{n}$. It is impossible to carry out the standard construction of the order complex counting such infinite objects. There is a more refined construction of conical resolutions, which helps us with some troubles of this kind (see, for example, Vassiliev 1999c,e; and $\S 3 a$ below), but in the case of knots this difficulty remains very serious.

Therefore, we restrict ourselves to the case of finite intersections: for any $d$ we consider only the poset $\Delta_{d}$ of planes (1.8) of codimension less than or equal to $n d$ in $\mathcal{K}$, construct the corresponding order complex, and define the general order complex $\Delta$ as the direct limit of such complexes over $d \rightarrow \infty$. The numbers $d$ define a natural increasing filtration on them. Any term $\phi_{d}$ of this filtration is finite dimensional and any difference $\phi_{d} \backslash \phi_{d-1}$ is naturally divided into a finite family of finite-dimensional cells, so that its one-point compactification is a finite cell complex.

The homological study of this filtered complex is a major problem in the theory of finite-type cohomology groups of knot spaces (and the theory of finite-type invariants is the part of this study considering only the cells of two upper dimensions in any term of the filtration).

Indeed, the resolved discriminant $\sigma \subset \Delta \times \mathcal{K}$ can be naturally projected to both $\Delta$ and $\mathcal{K}$. The first projection induces a natural filtration $F_{1} \subset F_{2} \subset \cdots$ on it from the filtration $\left\{\phi_{d}\right\}$ on $\Delta$. The restriction of this projection to the difference $F_{d} \backslash F_{d-1}$ is 

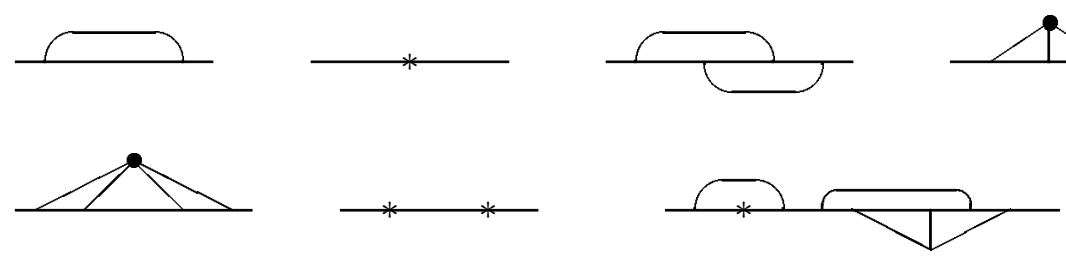

Figure 4. Examples of generalized chord diagrams.

a locally trivial bundle over $\phi_{d} \backslash \phi_{d-1}$ whose fibres are subspaces of codimension $n d$ in $\mathcal{K}$. Thus, the 'Borel-Moore homology group of finite codimension' of $F_{d} \backslash F_{d-1}$ is reduced (via some sort of the Thom isomorphism) to the usual (finite-dimensional) homology group of the base (which, in particular, is finitely generated). This allows us to calculate, in principle, all the (finite-codimension) homology groups of spaces $F_{d}$. The finite-type homology classes of $\Sigma$ are nothing else than direct images of their elements under the second projection $\sigma \rightarrow \Sigma$, and the finite-type cohomology classes of the space of knots are their Alexander duals. The 'order' (i.e. the filtration) of these classes is defined by our filtration of the resolved discriminant.

The cellular structure of terms $\phi_{d} \backslash \phi_{d-1}$ (and hence also of $F_{d} \backslash F_{d-1}$ ) together with incidence coefficients of cells is explicitly described in Vassiliev $(1990,1997)$. It consists of the enumeration of different families of planes (1.8) and the simplicial structure of inserted order complexes. $\dagger$

The families of planes (1.8) are classified and depicted in the terms of (generalized) chord diagrams (see figure 4). Any particular plane $L(a, b)$ is depicted by an arc (chord) connecting the points $a, b$ of the line $\mathbb{R}^{1}$ or the circle $S^{1}$, and finite collections of such planes (giving planes (1.8) as their intersections) by collections of such chords or more complicated objects. For instance, seven pictures of figure 4 denote the following planes respectively: a plane $L(a, b), a \neq b$; a plane $L(a, a)$; a plane $L(a, c) \cap$ $L(b, d)$, where $a<b<c<d \in \mathbb{R}^{1}$; a plane $L(a, b) \cap L(b, c) \equiv L(b, c) \cap L(c, a) \equiv$ $L(c, a) \cap L(a, b)$, where $a<b<c$; a plane of codimension $3 n$ consisting of maps gluing together some fixed four points of $\mathbb{R}^{1}$; a plane $L(a, a) \cap L(b, b)$; and a certain plane of codimension $5 n$.

The order complex arising over the plane of the third type is just a segment (or, more precisely, the union of two segments joining the vertex corresponding to the plane $L(a, c) \cap L(b, d)$ with two vertices corresponding to planes $L(a, c)$ and $L(b, d)$, cf. figure $2 a, e)$. The order complex arising over the fourth picture coincides with that shown in figure $2 d$. The order complex over the fifth picture is two dimensional and is equal to the cone over the graph given in the lower part of figure 5 (not containing the segments with endpoint (1234)). Figure 5 presents the corresponding poset (more precisely, only its primitive edges).

The theory of these resolutions is related very much to the graph theory. For instance, let us resolve in the naive way the stratum of $\Sigma$ consisting of maps $\mathbb{R}^{1} \rightarrow \mathbb{R}^{n}$ with a unique $k$-fold selfintersection point. This stratum consists of intersections of

$$
\left(\begin{array}{l}
k \\
2
\end{array}\right)
$$

$\dagger$ In Vassiliev (1990), I have used the 'naive' resolution as in (c) in figure 2. In this (equivalent) approach the study of inserted order complexes is replaced by the study of inserted simplices reduced modulo their subcomplexes lying in the lower terms of the filtration (see Vassiliev 1993). 


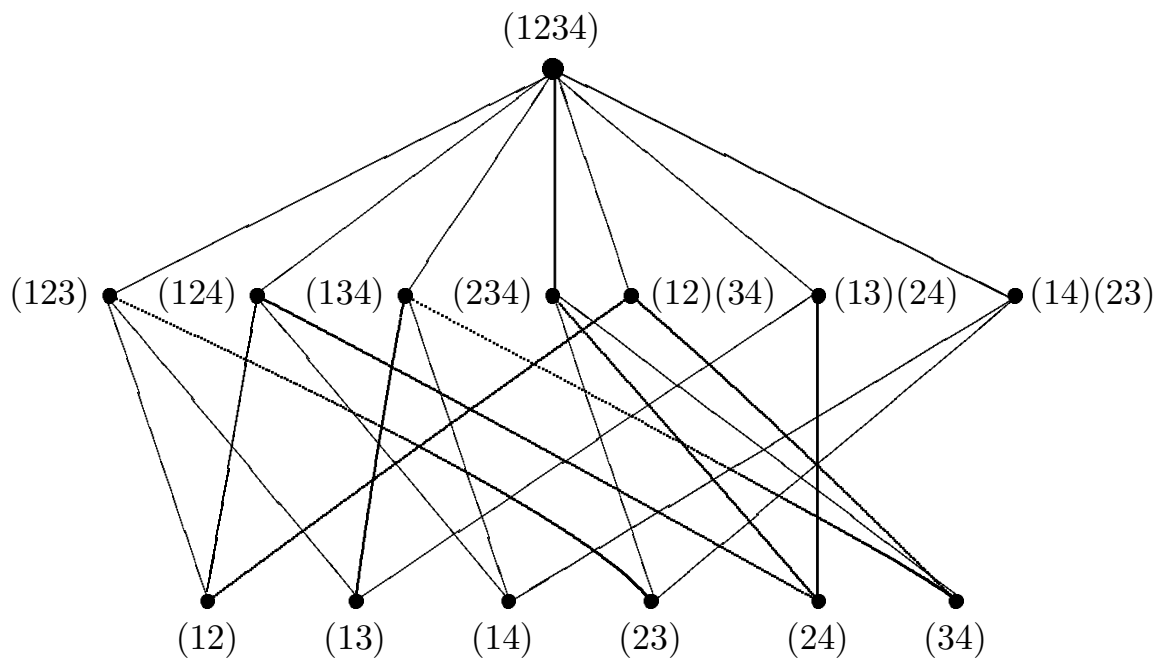

Figure 5. Poset and order complex for a quadruple point.

planes $L\left(a_{i}, a_{j}\right), 1 \leqslant i<j \leqslant k$. These planes correspond to the vertices of the inserted simplex. They are conveniently described by the edges connecting some pairs $(i, j)$ of $k$ numbered points, while the faces of this simplex are the collections of such edges, i.e. just the graphs on these $k$ vertices (without double edges or loops). Some of these faces belong to the lower term of our filtration of the discriminant: they are exactly the faces corresponding to the non-connected graphs. Therefore, we obtain the complex of connected graphs (see Vassiliev 1990, 1993). This complex arises also in the naive resolution of the 'diagonal' plane arrangement in $\mathbb{R}^{k n}$ consisting of all ordered collections of $k$ points in $\mathbb{R}^{n}$, at least two of which coincide. Another important related complex is that of two-connected graphs (see Babson et al. 1999; Turchin 1997, 1998; Vassiliev 1999a). On the other hand, the order complex arising from the economical resolution of the same stratum leads (after combining together some simplices) to the graph-complex of trees due to Kontsevich.

For the study of knot invariants in $\mathbb{R}^{3}$, it is enough to consider only the simplest chord diagrams like the ones in the first and the third pictures of figure 4, i.e. with all different endpoints. More precisely, such chord diagrams (and the corresponding cells) generate the homology groups responsible for knot invariants, while the relations between them are described in the terms of similar diagrams allowing either one asterisk, as in the second picture, or one triple point, as in the fourth.

However, for the calculation of higher cohomology groups of spaces of knots, the consideration of more complicated diagrams (such as the fifth and the last diagrams) is absolutely necessary.

\section{(g) The spectral sequence and its convergence}

The calculation of homology groups (of finite filtration) of the resolved discriminant (or of the cohomology classes in $\mathcal{K} \backslash \Sigma$ Alexander dual to them) can be presented by a cohomological spectral sequence with the support in the second quadrant (see 


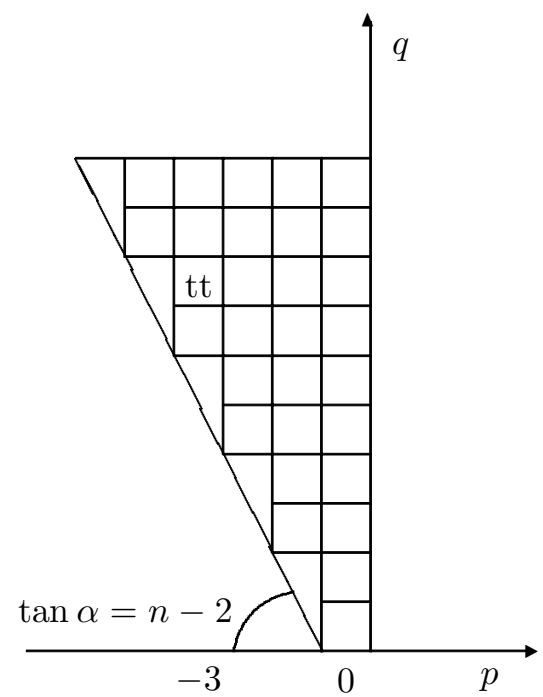

Figure 6. The spectral sequence.

figure 6). Its initial term $E_{1}$ is given by

$$
E_{1}^{p, q} \simeq \bar{H}_{n \infty-p-q-1}\left(F_{-p} \backslash F_{-p-1}\right) \simeq \bar{H}_{p(n+1)-q-1}\left(\phi_{-p} \backslash \phi_{-p-1}, A\right) .
$$

Here, $A$ is the orientation sheaf of the $n(\infty+p)$-dimensional affine bundle $\left(F_{-p} \backslash\right.$ $\left.F_{-p-1}\right) \rightarrow\left(\phi_{-p} \backslash \phi_{-p-1}\right)$. If $n$ is even, then this sheaf is isomorphic to $\mathbb{Z}$ (as the bundle is orientable), but if $n$ is odd, it generally is not. The order complexes $\phi_{d}$ do not depend on $n$, thus for different numbers $n$ of the same parities, the columns $E_{1}^{p, *}$ of spectral sequences calculating the cohomology of spaces of knots in $\mathbb{R}^{n}$ coincide canonically up to a shift along the $q$-axis. In the case of $\mathbb{Z}_{2}$-coefficients, the same is also true for $n$ of different parities.

If $n$ is greater than 3, then there are only finitely many non-zero cells on any diagonal $\{p+q=$ const. $\}$. Using the machinery of finite-dimensional approximations, it is easy to prove that, in this case, the infinitely degenerate strata of $\Sigma$ do not contribute to the calculation of cohomology classes. Therefore, if $n \geqslant 4$, then our spectral sequence converges exactly to the cohomology group $H^{*}(\mathcal{K} \backslash \Sigma)$ of the space of knots in $\mathbb{R}^{n}$.

For the most intriguing situation, $n=3$, this is not the case (or at least is not proved). Something good can be said, a priori, on the lower diagonal $\{p+q=0\}$ responsible for the knot invariants: any non-zero element of the group $E_{\infty}^{-i, i}$ actually defines a non-trivial knot invariant of filtration $i$ (modulo the group of invariants of smaller filtration). This filtration has a transparent geometrical description in the terms of finite differences (see Birman \& Lin 1993; Bar-Natan 1995; or $\S 0.2$ in Vassiliev 1990). However, for the elements of terms $E_{\infty}^{p, q}$ on higher diagonals, it is not known whether the infinitely degenerate strata will not spoil them. Any such element defines a $(p+q)$-dimensional cohomology class of $\mathcal{K} \backslash \Sigma$ (again, modulo the elements of lower filtration), but we cannot be sure, a priori, that this class is not trivial, i.e. that the corresponding cycle in the discriminant is not a boundary. 


\section{(h) Justifications and approximations}

Using the Weierstrass approximation theorem, we can choose a perfect (in some sense) system of finite-dimensional affine approximating subspaces $\left\{\mathcal{K}_{\nu}\right\}, \nu \rightarrow \infty$, of the space of curves $\mathcal{K}$. The corresponding rings $H^{*}\left(\mathcal{K}_{\nu} \backslash \Sigma\right)$ converge to the ring $H^{*}(\mathcal{K} \backslash \Sigma)$. Also, we can assume that all planes $\mathcal{K}_{\nu}$ are in general position and are particularly transversal to the natural stratification of $\Sigma$. Then, for any $\nu$, the cohomological spectral sequence calculating $H^{*}\left(\mathcal{K}_{\nu} \backslash \Sigma\right)$ and constructed from the simplicial resolution of $\Sigma \cap \mathcal{K}_{\nu}$ also resembles figure 6 (although it will have only finitely many non-trivial columns). The stabilization of spectral sequences means the following: for any natural $s$, there exists $\nu$ such that terms $E_{1}^{p, q}, p \geqslant-s$, of all our spectral sequences calculating cohomology of $\mathcal{K}_{\nu} \backslash \Sigma, \mathcal{K}_{\nu+1} \backslash \Sigma$, etc., are canonically isomorphic, and the images of differentials $d^{r}: E_{r}^{p, q} \rightarrow E_{r}^{p+r, q-r+1}$, $r \leqslant s$, acting from these cells to the right, also coincide. Thus, the limit spectral sequence $E_{r}^{p, q} \equiv \lim _{\nu \rightarrow \infty} E_{r}^{p, q}(\nu)$ is well defined.

A very important role in the birth of this theory was played by Arnold's problem concerning the stable cohomology ring of complements of discriminants of complex hypersurface singularities (see Arnold 1976). Still being of a finite-dimensional nature, this problem forced me to look for the homology classes arising uniformly in 'very high-dimensional' discriminant varieties, and also to think about the nature of their stabilization (see Vassiliev 1987).

\section{Further results and problems}

\section{(a) Kontsevich integral}

Theorem 2.1. For any $n \geqslant 3$, our spectral sequence with complex coefficients stabilizes at the first term:

$$
E_{\infty / \mathbb{C}}^{p, q} \simeq E_{1 / \mathbb{C}}^{p, q}
$$

This theorem was proved by Kontsevich and is surely true. Its published part proves the stabilization of the diagonal responsible for knot invariants, i.e. the equality (2.1) for $n=3$ and $p+q=0$ (see Chmutov \& Duzhin 2001; Kontsevich 1993a). For an arbitrary $n \geqslant 3$, almost the same integral proves the identity (2.1) on the lower boundary of the spectral sequence, i.e. for cells $E^{p, q}$ with $q+(n-2) p=0$. However, for the upper cells, the proof uses some extra effort.

A great problem is whether the same is true over the integers.

I conjecture that in the case of long knots this is true, and, moreover, that the homotopy splitting (1.5) of any finite term $\bar{F}_{d}$ of the filtration of the one-point compactification of our resolved discriminant holds in some precise sense (see, for example, Vassiliev 1999b).

Another great achievement coming from Kontsevich's works is an integral representation of the cohomology classes.

A spectral sequence similar to (but easier than) the one outlined in $\S \S 1 d, e$ allows us to calculate the cohomology groups of spaces $Y^{X}$ of continuous maps $X \rightarrow Y$, where $X$ is an $m$-dimensional finite cell complex and $Y$ an $m$-connected one. Indeed, $Y$ is homotopy equivalent to the space $\mathbb{R}^{N} \backslash \Lambda$, where $N$ is sufficiently large and $\Lambda$ is a closed conical subset of codimension greater than or equal to $m+2$. Then we consider the vector space of all continuous maps $X \rightarrow \mathbb{R}^{N}$, define the discriminant 

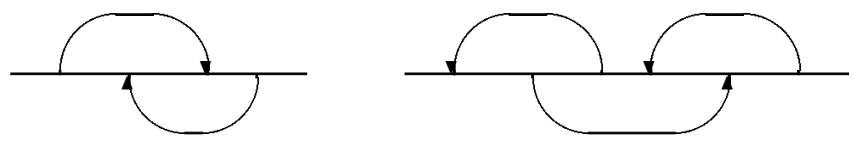

Figure 7. Arrow diagrams.

in it as the space of all maps whose images intersect $\Lambda$, resolve this discriminant as previously, and obtain a spectral sequence converging to the cohomology group of the complement of this discriminant, i.e. of the space $\left(\mathbb{R}^{N} \backslash \Lambda\right)^{X} \sim Y^{X}$ (see Vassiliev 1994a, 1997). This spectral sequence coincides with Anderson's spectral sequence (Anderson 1972) in the case when $X$ is a smooth manifold. It also provides some information if $Y$ is only $(m-1)$-connected, but in this case we cannot be sure that it calculates all the cohomology groups of $Y^{X}$, in full analogy with the discussion at the end of $\S 1 \mathrm{~g}$. If $X$ is a circle, then it is covered by the Adams-Eilenberg-Moore spectral sequence (Adams 1957; Eilenberg \& Moore 1966) (particularly calculating the cohomology of loop spaces). The 'deRhamization' of the latter spectral sequence is known as the theory of iterated path integrals (see Chen 1977; Hain 1984). Kontsevich's integral (and possibly also its smarter versions proving theorem 2.1 in full generality) can be considered as its extension to the problems 'of second order' (see $\S 2 d$ below).

\section{(b) Combinatorial expressions}

The most well-studied part of this theory is, of course, that of knot invariants. Shortly after its appearance, different combinatorial formulae for these invariants were developed. They express the values of invariants in the terms of the geometrical disposition of the knot (see, for example, Cartier 1993; Lannes 1993; Piunikhin 1995). The most convenient formulae of this kind were announced and partly proved by Polyak \& Viro (1994, 1996) (see also Tyurina 1999a,b).

These formulae are described in terms of arrow diagrams, i.e. pictures like the ones shown in figure 7 .

Let us fix a direction in $\mathbb{R}^{3}$ transverse to the common direction 'at infinity' of our long knots. Given a generic long knot $f: \mathbb{R}^{1} \rightarrow \mathbb{R}^{3}$ (see figure 1 ), the value on it of the left picture in figure 7 is equal to the number of 4 -configurations $(a<b<c<d) \subset \mathbb{R}^{1}$ (counted with appropriate signs), such that the point $f(a)$ lies above $f(c)$ with respect to the chosen direction, and $f(d)$ lies above $f(b)$. It turns out (see Polyak \& Viro 1994) that this value is actually a knot invariant, namely, it coincides with the unique invariant of filtration 2 .

Goussarov has proved a wonderful theorem as follows.

Theorem 2.2 (see Goussarov et al. 2000). Any invariant of finite filtration of long knots in $\mathbb{R}^{3}$ can be represented by a finite linear combination of arrow diagrams.

Formally speaking, any cohomology class of finite filtration of the space of knots in $\mathbb{R}^{n}, n \geqslant 3$, should also have combinatorial representations, although it may be formulated in terms of more complicated conditions whose total complexity is difficult to estimate. The strength of the previous theorem lies in the fact that, in the case of invariants, it is sufficient to use conditions of a very special kind. To find the combinatorial formulae for other cohomology classes $\alpha \in H^{*}(\mathcal{K} \backslash \Sigma)$ effectively, it is convenient to consider a combinatorial formula such as a semialgebraic relative 
cycle in $\mathcal{K}(\bmod \Sigma)$, such that $\alpha$ equals the linking number with the boundary of this cycle in $\Sigma$.

It is natural to construct such cycles by induction over our spectral sequence. For an illustration, let us consider again the theory of plane arrangements and their complements. In the case of the line arrangement shown in figure $2 c$, the entire group $E_{2, *}^{1}$ appears from the unique crossing point $L_{(12)}$. This group is non-trivial only for $*=-1$, is isomorphic to $\mathbb{Z}$ and is generated by the homology class of the segment $\Delta(1,2)$ modulo its endpoints (lying in $F_{1}$ ). The splitting formula (1.5) means that we can extend this relative cycle in $\bar{F}_{2}\left(\bmod \bar{F}_{1}\right)$ to a (Borel-Moore) cycle in entire $\tilde{L}$. However, to be able to define the value of this generating element on any 0 dimensional cycle in $\mathbb{R}^{2} \backslash L$, we need to choose such an extension explicitly. Then we project it to $L$ and get a cycle there. Finally, we need to choose a relative cycle in $\mathbb{R}^{2}(\bmod L)$ whose boundary coincides with this cycle. Then we call this relative cycle 'a combinatorial formula': its value on a point in $\mathbb{R}^{2} \backslash L$ is equal to the multiplicity of this cycle in the neighbourhood of this point.

If we have a more complicated plane arrangement, then we can construct this extension step by step over our filtration. Our starting element $\gamma \in E_{p, q}^{1}$ is represented by a cycle with closed supports in $F_{p} \backslash F_{p-1}$ (or, equivalently, by a relative cycle in $\left.\bar{F}_{p} / \bar{F}_{p-1}\right)$. We take its first boundary $d_{1}(\gamma)$, which is a cycle in $F_{p-1} \backslash F_{p-2}$. Then we span it, i.e. construct a chain $\tilde{\gamma}_{1} \subset F_{p-1} \backslash F_{p-2}$, such that $\partial \tilde{\gamma}_{1}=d_{1}(\gamma)$ there. Then we take the boundary of $\gamma+\tilde{\gamma}_{1}$ in the space $F_{p-2} \backslash F_{p-3}$ and span it there by a chain $\tilde{\gamma}_{2}$, etc. The splitting formula (1.5) ensures that this entire sequence of choices can be accomplished. Moreover, a precise final result of this sequence has been known since Ziegler \& Živaljević (1993) (see $\S 1 d$ ). It appears if we span our cycles in the most obvious way: by the trajectories of generic flows.

The case of knots (say, of long knots) is very similar to that of plane arrangements. For instance, here is a heuristic interpretation of the Polyak-Viro arrow diagram formulae. A knot invariant can be considered as a relative cycle of full dimension $n \infty$ in the space of curves $\mathcal{K}(\bmod \Sigma)$. Its value at a knot $f$ equals the multiplicity of the cycle in a neighbourhood of $f$ in $\mathcal{K}$. All strata of the discriminant which can generate (finite-type) homology classes of this dimension are defined only by ordinary chord diagrams. All points $a_{i}, b_{i}$ in (1.8) should be different. At them, the corresponding planes $L\left(a_{i}, b_{i}\right)$ meet normally, so that the corresponding order subcomplexes $\Delta(\cdot)$ are simplices (or, more precisely, their first barycentric subdivisions). I do not know a suitable analogue of a globally defined vector field $V$ from $\S 1 d$ on the space $\mathcal{K}$. However, in the construction of $\S 1 d$ we could use not the one vector $V$, but just a generic family of such vectors, one for each stratum $L_{I}$, whose trajectories span them in greater strata. In the case of knots, when the planes $L\left(a_{i}, b_{i}\right)$ in (1.8) are defined by conditions $f\left(a_{i}\right)=f\left(b_{i}\right)$, it is natural to take a vector field preserving the projection of our knot to $\mathbb{R}^{2}$ but increasing all the differences $z\left(b_{i}\right)-z\left(a_{i}\right)$, where $z$ is some coordinate in $\mathbb{R}^{3}$, say, the one normal to the 'blackboard' plane $\mathbb{R}^{2}$. To make this formula correct, we need to order the endpoints of any chord, i.e. to call one of them $a_{i}$ and the other $b_{i}$. Thus, the arrow diagrams appear. The union of wedges emanating from the point $f$, as in $\S 1 d$, will then consist of curves with the same projection to $\mathbb{R}^{2}$ but with $f\left(b_{i}\right)$ 'above' $f\left(a_{i}\right)$. The knot theory is very nonlinear (in contrast to the theory of plane arrangements). Particularly, such wedges corresponding to chord diagrams of the same topological type but with different configurations of points $a_{i}$, $b_{i}$ can have intersections in $\mathcal{K}$. The algebraic multiplicity of such an intersection at 


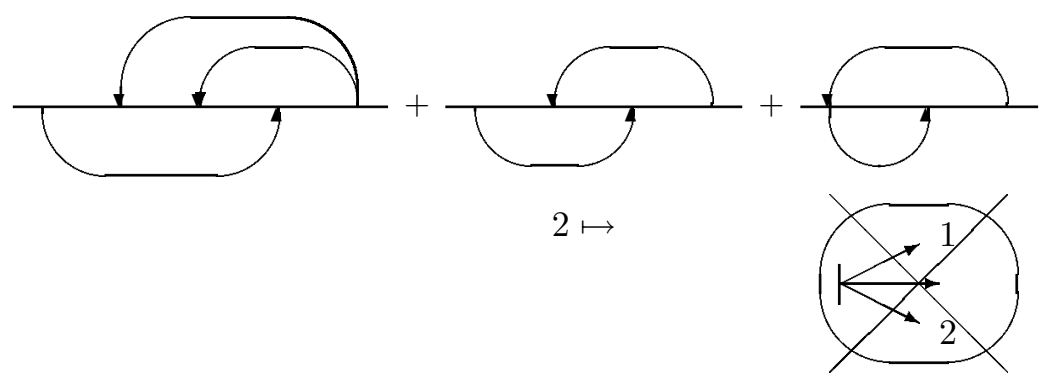

Figure 8. Combinatorial formula for Teiblum-Turchin cocycle.

some point $f \in \mathcal{K} \backslash \Sigma$ is exactly the value (in the Polyak-Viro sense) of the arrow diagram on the corresponding knot.

Of course, not everything is so easy. Indeed, the strata corresponding to different chord diagrams have common boundaries as the endpoints of different chords tend to one another. Some additional trouble comes from singular maps with nongeneric projections to $\mathbb{R}^{2}$. Therefore, the wedges constructed as above have some extra boundary components. Constructing the combinatorial formulae, we need to span these boundaries by some other chains in $\mathcal{K}$ or try to choose the orientations of arrows in such a way that these boundaries of different wedges annihilate. Goussarov's theorem means (in our terms) that it is always possible to choose the orientations of arrows in such a way that, for the spanning chain, we can take sums of similar wedges emanating from the strata (1.8) of lower complexity.

The above heuristic speculations are also helpful in the case of higher dimensions (in any of the senses indicated in the abstract), i.e. in constructing the combinatorial expressions of higher-dimensional cohomology classes of spaces of knots in $\mathbb{R}^{n}, n \geqslant 3$.

In Vassiliev (2001), natural classes of semialgebraic subvarieties in $\mathcal{K}$ and in different terms, $F_{i} \backslash F_{i-1}$, of the filtration were introduced, of which (some of) these spanning chains can be built.

\section{(i) Example: Teiblum-Turchin cocycle and its realization}

The first positive-dimensional cohomology class of finite filtration of the space of long knots in $\mathbb{R}^{3}$ was calculated by my students (D. M. Teiblum \& V. E. Turchin 1995, unpublished work; Vassiliev 1997, 1999b). It is a class of dimension 1 and filtration 3. (According to Vassiliev (1990), there are no cohomology classes of filtration less than or equal to 2, other than the simplest knot invariant.) However, this calculation was quite implicit: they calculated just the corresponding group $E_{1}^{-3,4} \sim \mathbb{Z}$ of the spectral sequence in the terms of generalized chord diagrams. It is clear from the shape of the spectral sequence that this group survives and that the final group $E_{\infty}^{-3,4}$ is also isomorphic to $\mathbb{Z}$, so that its generator can be extended to a well-defined one-dimensional cohomology class of the space of knots.

However, the fact that this class is non-trivial does not follow from the general considerations (see the discussion in $\S 1 \mathrm{~g}$ ). This fact was proved in Vassiliev (2001) by means of an explicit combinatorial formula (see figure 8 and the following theorem).

Let us choose a direction 'to the right' in the 'blackboard' plane $\mathbb{R}^{2}$ (i.e. in the quotient of the space $\mathbb{R}^{3}$ by the direction chosen previously). 
Theorem 2.3 (see Vassiliev 2001). The value of the Teiblum-Turchin cocycle on any generic loop in the space $\mathcal{K}$ of long knots (i.e. on a closed 1-parametric family of such knots) is equal mod 2 to the number of points of this loop such that one of the following three conditions holds (see figure 8).

(i) There are five points $a<b<c<d<e$ in $\mathbb{R}^{1}$ such that $f(a)$ is above $f(d)$, and $f(e)$ is above $f(c)$ and $f(b)$.

(ii) There are four points $a<b<c<d$ in $\mathbb{R}^{1}$ such that $f(a)$ is above $f(c), f(b)$ is below $f(d)$, and the projection of the derivative $f^{\prime}(b)$ to $\mathbb{R}^{2}$ is directed to the right.

(iii) There are three points $a<b<c$ in $\mathbb{R}^{1}$ such that $f(a)$ is above $f(b)$ but below $f(c)$, and the 'exterior' angle in $\mathbb{R}^{2}$ formed by projections of $f^{\prime}(a)$ and $f^{\prime}(b)$ contains the direction 'to the right' (i.e. this direction is equal to a linear combination of these projections, and at least one of the coefficients in this combination is non-positive).

(These points of the loop in $\mathcal{K}$ should be counted with multiplicities equal to the numbers of different point configurations in $\mathbb{R}^{1}$ for which the corresponding condition (i), (ii) or (iii) is satisfied.)

This statement remains true if we replace $\mathbb{R}^{3}$ by any $\mathbb{R}^{n}, n \geqslant 3, \mathbb{R}^{2}$ by $\mathbb{R}^{n-1}$, a generic loop in the space of knots by a generic $(3 n-8)$-dimensional cycle, and the one-dimensional Teiblum-Turchin cocycle by its $(3 n-8)$-dimensional stabilization (see discussion in $\S 1 \mathrm{~g}$ ).

Further, let us consider the connected sum of two equal (long) trefoil knots in $\mathbb{R}^{3}$ and a path in the space of knots connecting this knot with itself, as in the proof of the commutativity of the knot semigroup. We shrink the first summand, move it 'through' the second, and then blow it up again.

Proposition 2.4. This closed path in the space of long knots has exactly seven intersection points (counted with multiplicities) with the union of three varieties indicated in items (i), (ii) and (iii) of the previous theorem.

However, the Teiblum-Turchin cocycle is a well-defined integral cohomology class, thus its value on (the integral homology class of) this loop is not equal to zero, and the group generated by this cocycle is free.

Remark 2.5. I cannot yet reprove the Goussarov's theorem in this way: the combinatorial formulae for knot invariants obtained by the straightforward application of our algorithm can include some varieties in $\mathcal{K}$ that are more complicated than the varieties just given by arrow diagrams (see theorem 2.3). The construction of spanning cycles participating in this algorithm leaves many choices, e.g. how to order the endpoints $a_{i}, b_{i}$ of a chord. Goussarov's theorem implies that it is possible to choose these possibilities in such a way that all the awkward varieties will be cancelled. I hope that a deeper understanding of its proof will help to formulate the exact rule for this.

Also, in all situations that are more complicated than that of invariants, I do not have, strictly speaking, an algorithm (i.e. something definitely converging to an answer), but just a collection of tricks which succeed in giving such answers to particular problems as that of the Teiblum-Turchin cocycle or as the one considered in the next subsection. 
Remark 2.6. The virtual knots introduced by L. Kauffman in 1997 and applied in Goussarov et al. (2000) to the construction of combinatorial formulae can be identified as another (extremely big) class of subvarieties of the space of curves $\mathcal{K}$.

\section{(c) Cohomology of spaces of compact knots}

A similar theory exists for the space of compact knots $S^{1} \rightarrow \mathbb{R}^{n}$. There is a oneto-one correspondence between invariants of compact and long knots in $\mathbb{R}^{3}$, but in higher dimensions, many extra cohomology classes of spaces of compact knots arise from the topological non-triviality of the circle. For instance, already in filtration 1 we have two such classes of dimensions $n-2$ and $n-1$ (with coefficients in $\mathbb{Z}_{2}$, and, if $n$ is even, also with integer coefficients). The combinatorial formulae for all such classes of filtrations 1 and 2 were found in Vassiliev (2001). For example, the $(n-2)$ dimensional class of filtration 1 is Alexander dual to the variety in $\mathcal{K}$ formed by all maps $f: S^{1} \rightarrow \mathbb{R}^{N}$ glueing together some two opposite points of $S^{1}$ (see Vassiliev $1999 a)$.

The corresponding combinatorial formula consists of two varieties distinguished by the following conditions (referring to a circular coordinate $S^{1} \sim \mathbb{R} / 2 \pi \mathbb{Z}$ in $S^{1}$ ):

(a) there is a point $\alpha \in(0, \pi)$ such that $f(\alpha)$ is above $f(\alpha+\pi)$ with respect to the chosen direction;

(b) the projection of the point $f(0)$ to $\mathbb{R}^{n-1}$ lies 'to the right' of the projection of $f(\pi)$.

As usual, this entire theory can be literally extended to the spaces of links, i.e. embeddings of a disjoint union of finitely many circles.

\section{(d) Theories of further orders}

The knot theory is a theory of the second degree of complexity in the same way as the problem mentioned at the end of $\S 2 a$ is of degree one. The forbidden discriminant set in the knot theory is defined by a condition on the simultaneous behaviour of our map $\mathbb{R}^{1} \rightarrow \mathbb{R}^{n}$ at some two points, while, in the theory of generalized loop spaces, any point is only responsible for its own behaviour.

The typical example of a problem of order 3 is the study of invariants of plane immersed curves $S^{1} \rightarrow \mathbb{R}^{2}$ without triple self-intersection points.

This problem was raised by Arnold (1994, 1995), who also indicated the simplest such invariant separating homotopic immersions. This is the strangeness Alexander dual to the fundamental cycle of the whole discriminant variety of curves having forbidden triple points.

Similarly to the case of knots, this variety is swept out by the 3-parametric family (parametrized by three-point configurations in $S^{1}$ ) of flat manifolds of codimension 4 in the entire space of plane curves. (These manifolds form open dense subsets in the planes that are also parametrized by triples of points and distinguished by the condition that the images of these three points should coincide.) It easily follows that this discriminant variety is the image of a smooth orientable manifold, and, in particular, carries a fundamental cycle. For further results on this classification see Shumakovich (1996) and Vassiliev (1999d). 
(a)

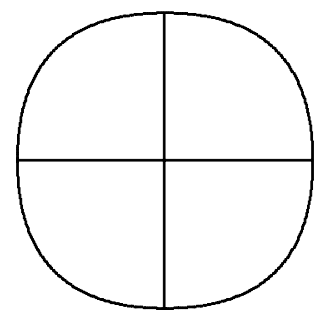

(b)

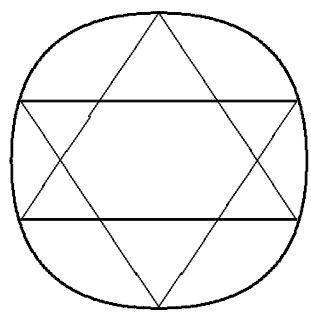

(c)

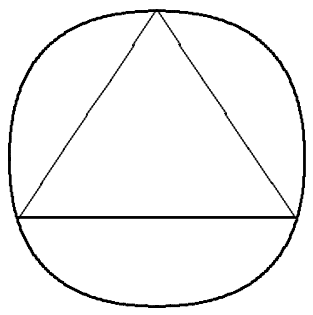

Figure 9. Simplest invariants of knots and doodles.

A similar problem formulated in Vassiliev $(1993,1994 b)$, and studied in Khovanov (1996), Merkov (1998, 1999) and Vassiliev (1999d) among others, concerns the classification of all smooth plane curves $S^{1} \rightarrow \mathbb{R}^{2}$ (not necessarily immersions) without triple points or singularities obtained as their degenerations. (Since Khovanov (1996), they are called doodles; for a similar problem see also Fenn \& Taylor (1977) and Fock et al. (1992))

These problems have lead to the calculus of triangular diagrams (see Vassiliev $1999 d$ ) in the same way as the knot theory leads to the chord algebra. For example, Arnold's 'strangeness' is an invariant of filtration 2 and can be depicted by a single triangle (see figure 9c). However, it is not an invariant of doodles. The simplest invariant of doodles (discovered first by Merkov (1998) by different methods) can be naturally depicted by the simplest triangular diagram, whose triangles have no neighbouring points in the circle (see figure $9 b$ ) in the same way as the first knot invariant corresponds to the simplest chord diagram with the same property (see figure $9 a$ or the third picture of figure 4 ).

The relation to the graph theory (see p. 1350) is almost literally replaced by the relation to the theory of 3-hypergraphs, and the analogy with the 'diagonal' plane arrangement is replaced by the analogy with the ' $k$-equal' arrangement of planes in $\mathbb{R}^{n m}=\left(\mathbb{R}^{n}\right)^{m}$ consisting of such collections $\left(x_{1}, \ldots, x_{m}\right), x_{i} \in \mathbb{R}^{n}$, that $x_{i_{1}}=\cdots=x_{i_{k}}$ for some set of indices $1 \leqslant i_{1}<\cdots<i_{k} \leqslant m$ (see Björner \& Welker 1995; Vassiliev 1993).

\section{(e) Turchin's calculation}

The theory of finite-type invariants of knots has borne many beautiful algebraic objects, such as the Hopf algebra of chord diagrams and the graph-complex of trees (see, for example, Bar-Natan 1995; Chmutov et al. 1998; Kontsevich 1993b).

It was shown recently in Tourtchine (2000) that these structures are non-separable parts of more general theories, related with entire cohomology rings of spaces of knots and formulated in terms of generalized chord diagrams. The corresponding multiplicative structures resemble the multiplication discussed in $\S 1 e$, although they are, of course, much more complicated. It was proved in Tourtchine (2000) that the first term of the main spectral sequence calculating the rational homology of the space of long knots in $\mathbb{R}^{n}, n \geqslant 3$, is described in terms of the Hochschild homology of the Poisson algebras operad if $n$ is odd (respectively, of the Gerstenhaber algebras operad, if $n$ is even). Namely, the Hochschild homology of these operads is, in both cases, some polynomial algebra in infinitely many even and odd variables. To obtain the first term of the spectral sequence in the case of even $n$, we need to factorize the 
corresponding polynomial algebra by one odd generator $\left[x_{1}, x_{2}\right]$. In the case of odd $n$, we need to factorize by two generators: one even (equal to $\left[x_{1}, x_{2}\right]$ ) and one odd (equal to $\left.\left[\left[x_{1}, x_{3}\right], x_{2}\right]\right)$.

In particular, the standard bialgebra of chord diagrams factorized through the 4-term relations (see Bar-Natan 1995; Kontsevich 1993a) is some subspace in the Hochschild homology of the Poisson algebras operad. To obtain the algebra of finiteorder invariants (i.e. cohomology of degree zero in the case $n=3$ ), we should factorize this bialgebra by one generator $\left[x_{1}, x_{2}\right]$.

\section{Discussion}

(a) Which method of resolution is better: the naive or the economical method (figure 2c, $d$ )?

This question was asked by Professor Hitchin at the Meeting. In the case of knot spaces they are more or less equal. The constructions are equivalent and the complexities of related calculations are comparable. However, generally, the 'economical' method (or rather its suitable generalization) is stronger. Indeed, sometimes we need to resolve discriminant spaces swept out by families of planes, infinitely many of which pass through one and the same point. The classical example is the determinant variety of all degenerate linear operators (whose Borel-Moore homology group is Alexander dual to the cohomology group of $G L_{n}$ ), or the space of singular algebraic projective hypersurfaces of a given degree (that arises in the study of the complementary space of non-singular varieties), etc. (see Vassiliev 1991, 1999c,e). The natural extension of the 'economical' construction based on the notions of conical resolutions and continuous order complexes allows us to overcome this difficulty, while the naive construction does not work.

On the other hand, sometimes using both methods together is better than any one used singly. This means that the very fact that both constructions are homotopy and homology equivalent provides interesting combinatorial relations. For instance, the resolutions of diagonal arrangements as in Arnold (1969) (arising also in the study of some discriminant strata of the space of knots) are related very much to the graph theory, and we obtain many natural problems and comparison theorems in its homological theory (see, for example, Babson et al. 1999; Björner \& Welker 1995; Turchin 1997, 1998; Vassiliev 1993, 1999a).

\section{(b) Caution}

The initial part of the described theory, i.e. the study of knot invariants of finite filtration, became very popular (see, for example, Bar-Natan 1994) owing, in particular, to the fact that its basic definitions can be formulated in very elementary terms of finite differences (see Bar-Natan 1995; Birman 1993; Birman \& Lin 1993; Vassiliev 1990, $\S \S 0.2,0.4)$.

However, the literal translation of these definitions to such problems as, for example, theories of higher orders in the sense of $\S 2 d$ or the study of higher-dimensional cohomology classes of spaces of knots will not help us to guess adequate geometrical constructions or equally beautiful algebraic structures. For instance, the group of 'order $k$ ' (in this sense) invariants of triple points free plane curves will not be finitely generated for any $k$. The reason for this consists of the fact that, in these 
theories, the singularities of discriminant sets that are essential for the calculation of cohomology classes and invariants are more complicated than just the normal crossings. Moreover, the indices that invariants and cohomology classes define at all such essential strata (by appropriate non-obvious analogues of conditions of type

$$
f(-\mid-)+f\left(\frac{1}{\mathrm{l}}\right)=f(-\downarrow)
$$

from the theory of knot invariants) are, in most situations, not scalar: they take values in certain homology groups associated with these strata (see Vassiliev 1999a). Therefore, the elementary interpretation of the filtration, as well as the very definition of objects of finite type, should be adequately modified in any particular theory of this sort (see Cattaneo et al. 2000; Merkov 1998; Vassiliev 1994b, 1999d).

Notice, however, the beautiful theory of finite-type invariants of 3-manifolds begun by Ohtsuki and extended by Garoufalidis, Goussarov and others (see Garoufalidis 1996; Goussarov 1999; Ohtsuki 1996). In this theory, some comparatively close modification of the basic geometrical characterization of knot invariants of finite filtration is very important. Maybe this can be explained by the fact that the classification of 3-manifolds is a (very non-trivial) quotient of the link theory by the Kirby relations.

Unfortunately, I cannot include this theory in the general framework of the discriminant theory.

I acknowledge the hospitality of the Isaac Newton Institute, Cambridge, where this article was written. During this work, I was helped by conversations and correspondence with A. B. Merkov, M. E. Kazarian, V. E. Turchin, M. de Longueville and C. Schultz. The research presented here was supported in part by an NWO grant (project 047-008-005).

\section{References}

Adams, J. F. 1957 On the cobar construction. In Colloque de topologie algébrique, Louvain, 1956, pp. 81-87. Paris: George Thone.

Anderson, D. W. 1972 A generalization of the Eilenberg-Moore spectral sequence. Bull. Am. Math. Soc. 78, 784-786.

Arnold, V. I. 1969 Cohomology ring of the group of dyed braids. Mat. Zametki (Math. Notes) 5, 227-231.

Arnold, V. I. 1970 On some topological invariants of algebraic functions. Trans. (Trudy) Moscow Mat. Soc. 21, 27-46.

Arnold, V. I. 1976 Some unsolved problems of the singularity theory. In Proc. of the S. L. Sobolev seminar, Mathematics Institute of the Siberian Branch of the Russian Academy of Science, Novosibirsk, Russia (ed. S. V. Uspenskii), pp. 5-15. (In Russian. Extended English translation: 1983 Singularities. Part 1 (Arcata 1981) (ed. P. Orlik), pp. 57-69. Providence, RI: American Mathematical Society.)

Arnold, V. I. 1989 Spaces of functions with mild singularities. Funct. Analysis Appl. 23, 1-10.

Arnold, V. I. 1994 Plane curves, their invariants, perestroikas and classifications. In Singularities and bifurcations (ed. V. I. Arnold), vol. 21, pp. 33-91. Advances in Soviet Mathematics. Providence, RI: American Mathematical Society.

Arnold, V. I. 1995 Invariants and perestroikas of fronts in the plane. Proc. Steklov Math. Inst. 209, 14-64.

Babson, E., Björner, A., Linusson, S., Shareshian, J. \& Welker, V. 1999 The complexes of not $i$-connected graphs. Topology 38, 271-299.

Bar-Natan, D. 1994 Bibliography of Vassiliev invariants. Available at http://www.ma.huji.ac.il/ $\sim$ drorbn/VasBib/VasBib.html. 
Bar-Natan, D. 1995 On the Vassiliev knot invariants. Topology 34, 423-472.

Birman, J. 1993 New points of view in knot theory. Bull. Am. Math. Soc. 28, 253-287.

Birman, J. S. \& Lin, X.-S. 1993 Knot polynomials and Vassiliev's invariants. Invent. Math. 111, $225-270$.

Björner, A. \& Welker, V. 1995 The homology of ' $k$-equal' manifolds and related partition lattices. Adv. Math. 110, 277-313.

Brieskorn, E. 1973 Sur les groupes de tresses (d'après V. I. Arnold). In Seminaire Bourbaki 1971, no. 401, pp. 21-44. Lecture Notes in Mathematics, vol. 317. Springer.

Cartier, P. 1993 Construction combinatoire des invariants de Vassiliev. C. R. Acad. Sci. Paris Sér. I Math. 316, 1205-1210.

Cattaneo, A. S., Cotta-Ramusino, P. \& Longoni, R. 2000 Configuration spaces and Vassiliev classes in any dimension. Preprint math.GT/9910139.

Chen, K. T. 1977 Iterated path integrals. Bull. Am. Math. Soc. 83, 831-879.

Chmutov, S. V. \& Duzhin, S. V. 2001 Kontsevich integral. Acta Applicandae Math. 66, 155-190.

Chmutov, S. V., Duzhin, S. V. \& Lando, S. V. 1994 Vassiliev knot invariants. II. Intersection graph conjecture for trees. In Singularities and curves (ed. V. I. Arnold), vol. 21, pp. 127-134. Advances in Soviet Mathematics. Providence, RI: American Mathematical Society.

Chmutov, S. V., Duzhin, S. V. \& Kaishev, A. I. 1998 The algebra of 3-graphs. Proc. Steklov Math. Inst. 221, 168-196.

De Concini, C. \& Procesi, C. 1995 Wonderful models of subspace arrangements. Sel. Math. New. Ser. 1, 459-494.

Deligne, P. 1972 Immeubles des groupes de tresses généralisées. Invent. Math. 17, 273-302.

Deligne, P., Goresky, M. \& MacPherson, R. 2000 L'algèbre de cohomologie du complément, dans un espace affine, d'une famille finie de sous-espaces affines. Michigan Math. J. 48, 121-136.

de Longueville, M. \& Schultz, C. A. 2001 The cohomology rings of complements of subspace arrangements. Math. Annln 319, 625-646.

Eilenberg, S. \& Moore, J. C. 1966 Homology and fibrations. I. Comment. Math. Helv. 40, 199236.

Feichtner, E. M. \& Ziegler, G. M. 2000 On cohomology algebras of complex subspace arrangements. Trans. Am. Math. Soc. 352, 3523-3555.

Fenn, R. \& Taylor, P. 1977 Introducing doodles. In Topology of low-dimensional manifolds (ed. R. Fenn), pp. 37-43. Lecture Notes in Mathematics, vol. 722. Springer.

Fock, V. V., Nekrasov, N. A., Rosly, A. A. \& Selivanov, K. G. 1992 What we think about the higher-dimensional Chern-Simons theories. In Sakharov memorial lectures in physics, vol. 1, pp. 465-471. Commack, NY: Nova.

Garoufalidis, S. 1996 On finite-type 3-manifold invariants. J. Knot Theory Ramificat. 5, 441-462.

Goresky, M. \& MacPherson, R. 1988 Stratified Morse theory. Springer.

Goussarov, M. 1999 Finite-type invariants and $n$-equivalence of 3-manifolds. C. R. Acad. Sci. Paris Sér. I Math. 329, 517-522.

Goussarov, M., Polyak, M. \& Viro, O. 2000 Finite-type invariants of classical and virtual knots. Topology 39, 1045-1068.

Hain, R. 1984 Iterated integrals and homotopy periods. Mem. Am. Math. Soc. 47, 291.

Khovanov, M. 1996 Doodle groups. Trans. Am. Math. Soc. 349, 2297-2315.

Kontsevich, M. $1993 a$ Vassiliev's knot invariants. In Advances in Soviet mathematics, vol. 16, ch. 2, pp. 137-150. Providence RI: American Mathematical Society.

Kontsevich, M. $1993 b$ Formal (non-)commutative symplectic geometry. In I. M. Gel'fand's mathematical seminars 1990-1992 (ed. L. Corvin, I. M. Gel'fand \& J. Lepovsky), pp. 173-187. Basel: Birkhäuser.

Lannes, J. 1993 Sur les invariants de Vassiliev de degré inferieur ou égal à 3. Enseign. Math. 39, 295-316. 
Merkov, A. B. 1998 Finite order invariants of ornaments. J. Math. Sci. 90, 2215-2273.

Merkov, A. B. 1998 Vassiliev invariants classify plane curves and doodles. Preprint available at http://www.botik.ru/ duzhin/as-papers/finv-dvi.zip.

Merkov, A. B. 1999 Vassiliev invariants classify flat braids. In Differential and symplectic topology of knots and curves (ed. S. L. Tabachnikov). Am. Math. Soc. Transl. 2 190, 83-102.

Merkov, A. B. 2001 Segment-arrow diagrams and invariants of ornaments. Mat. Sb. 191, 16351666.

Ohtsuki, T. 1996 Finite-type invariants of integral homology 3-spheres. J. Knot Theory Ramificat. 5, 101-115.

Orlik, P. \& Solomon, L. 1980 Combinatorics and topology of complements of hyperplanes. Invent. Math. 56, 167-189.

Piunikhin, S. A. 1995 Combinatorial expression for universal Vassiliev link invariant. Commun. Math. Phys. 168, 1-22.

Polyak, M. \& Viro, O. 1994 Gauss diagram formulas for Vassiliev invariants. Int. Math. Res. Notices 11, 445-453.

Polyak, M. \& Viro, O. 1996 On the Casson knot invariant. Tel Aviv University and Uppsala University. Preprint math.GT/9903158.

Rybnikov, G. L. 1998 On the fundamental group of the complex hyperplane arrangement. Preprint math.AG/9805056.

Shumakovich, A. 1996 Explicit formulas for strangeness of plane curves. St Petersburg Math. J. 7, 445-472.

Tourtchine, V. 2000 Sur l'homologie des espaces des nœuds non-compacts. ArXiv: math.QA/ 0010017.

Turchin, V. E. 1997 Homology groups of complexes of two-connected graphs. Uspekhi Math. Nauk 52, 189-190. (English Translation: Russ. Math. Surv. 52, 426-427.)

Turchin, V. E. 1998 Homology isomorphism of the complex of 2-connected graphs and the graph complex of trees. In Topics in quantum groups and finite-type invariants. Mathematics at the Independent University of Moscow (ed. B. Feigin \& V. Vassiliev). Am. Math. Soc. Transl. 2 185, 145-153.

Tyurina, S. D. $1999 a$ On the Lannes and Viro-Polyak type formulas for finite-type invariants. Matem. Zametki 66, 635-640. (English translation: Math. Notes 66, 525-530.)

Tyurina, S. D. $1999 b$ Diagrammatic formulas of the Viro-Polyak type for finite-degree invariants. Russ. Math. Surv. 54, 658-659.

Vassiliev, V. A. 1987 Stable cohomology of complements of discriminant manifolds of singularities of holomorphic functions. Russ. Math. Surv. 42, 307-308.

Vassiliev, V. A. 1990 Cohomology of knot spaces. In Theory of singularities and its applications (ed. V. I. Arnold), vol. 1, pp. 23-69. Advances in Soviet Mathematics. Providence, RI: American Mathematical Society.

Vassiliev, V. A. 1991 A geometric realization of the homology of classical Lie groups, and complexes, $S$-dual to the flag manifolds. Algebra i Analiz 3, 113-120. (English transl. St Petersburg Math. J. 3, 809-815.)

Vassiliev, V. A. 1993 Complexes of connected graphs. In I. M. Gel'fand's mathematical seminars 1990-1992 (ed. L. Corvin, I. M. Gel'fand \& J. Lepovsky), pp. 223-235. Basel: Birkhäuser.

Vassiliev, V. A. 1994 a Complements of discriminants of smooth maps: topology and applications, revised edn. Translations of mathematical monographs. Providence, RI: American Mathematical Society.

Vassiliev, V. A. $1994 b$ Invariants of ornaments. In Singularities and bifurcations (ed. V. I. Arnold), vol. 21, pp. 225-262. Advances in Soviet Mathematics. Providence, RI: American Mathematical Society.

Vassiliev, V. A. 1997 Topology of complements of discriminants. Moscow: Phasis. (In Russian.)

Phil. Trans. R. Soc. Lond. A (2001) 
Vassiliev, V. A. 1999a Topology of two-connected graphs and homology of spaces of knots. In Differential and symplectic topology of knots and curves (ed. S. L. Tabachnikov). Am. Math. Soc. Transl. 2 190, 253-286.

Vassiliev, V. A. $1999 b$ Homology of $i$-connected graphs and invariants of knots, plane arrangements, etc. In Proc. Arnoldfest Conf., Fields Institute Communications, vol. 24, pp. 451-469. Providence, RI: American Mathematical Society.

Vassiliev, V. A. 1999c How to calculate homology groups of spaces of non-singular algebraic projective hypersurfaces. Proc. Steklov Math. Inst. 225, 121-140.

Vassiliev, V. A. 1999d On finite-order invariants of triple points free plane curves. In D. B. Fuchs anniversary volume (ed. A. Astashkevich \& S. Tabachnikov). Am. Math. Soc. Transl. 2 194, $275-300$.

Vassiliev, V. A. 1999e Topological order complexes and resolutions of discriminant sets. Publ. Inst. Math. Belgrade Nlle Ser. 66, 165-185.

Vassiliev, V. A. 2001 On combinatorial formulas for cohomology of spaces of knots. Moscow Math. J. 1, 91-123.

Yuzvinsky, S. 1998 Small rational model of subspace complement. AMS Translations. Providence, RI: American Mathematical Society. Preprint available at http://xxx.lanl.gov/ abs/math.CO/9806143.

Yuzvinsky, S. 1999 Rational model of subspace complement on atomic complex. Publ. Inst. Math. Belgrade Nlle Ser. 66, 157-164.

Ziegler, G. M. \& Živaljević, R. T. 1993 Homotopy type of arrangements via diagrams of spaces. Math. Annln 295, 527-548. 